\title{
Araña roja en yaca: bioecología y eficacia biológica de acaricidas
}

\author{
Luis Martín Hernández Fuentes ${ }^{1 \S}$ \\ Efigenia Montalvo González ${ }^{2}$ \\ Yolanda Nolasco Gonzalez ${ }^{1}$ \\ Porfirio Gutiérrez Martínez ${ }^{2}$ \\ Héctor González Hernández ${ }^{3}$ \\ José Joaquín Velázquez Monreal ${ }^{1}$
}

${ }^{1}$ INIFAP. Progreso \#5, Barrio de Santa Catarina, Del. Coyoacán, Cd. de México. CP. 04010. Tel. 01 (800) 0882222, ext. 84406. (nolasco.yolanda@inifap.gob.mx; velazquez.josejoaquin@inifap.gob.mx). ${ }^{2}$ Instituto Tecnológico de Tepic. Avenida Tecnológico \#2595, Lagos del Country, Tepic, Nayarit. CP. 63175. Tel. 311 2119400. (efimontalvo@gmail.com; pgutierrez@ittepic.mx). ${ }^{3}$ Colegio de Postgraduados-Campus Montecillo. Carretera México-Texcoco km 36.5, Texcoco, Estado de México. CP. 56230. Tel. 5959520200. (hector.agave@hotmail.com).

${ }^{\S}$ Autor para correspondencia: hernandez.luismartin@inifap.gob.mx.

\section{Resumen}

La yaca (Artocarpus heterophyllus) se ha convertido en un frutal importante y representa una opción de producción para zonas tropicales de México. No obstante, por su reciente introducción, la información sobre sus plagas y enfermedades asociadas y recomendaciones de manejo es aún limitada. En 2017 se observó deformación de hojas, decoloración del haz y presencia de telaraña en el envés causado por un ácaro desconocido. El objetivo de esta investigación fue identificar el ácaro asociado, determinar su distribución, fluctuación poblacional y evaluación de eficacia biológica de acaricidas. El estudio se realizó durante 2018-2019 en Nayarit, México. El ácaro identificado es Tetranychus pacificus conocido como araña roja. Se distribuye en todas las zonas productoras de yaca en Nayarit. Se detectó de noviembre a junio con mayor infestación en la zona productora de Tecuitata ( 0.86 ácaros hoja ${ }^{-1}$ ) y Jalcocotan ( 0.57 ácaros hoja $^{-1}$ ) del municipio de San Blas. En promedio en septiembre y octubre es imperceptible su presencia; no obstante, en El Capomo sólo se detectó en julio lo que indica una etapa inicial de dispersión del ácaro. Se observó correlación significativa $(\beta=0.0489, r=-0.73, \mathrm{~F}=0.0345, \alpha=0.05)$ de la población con la temperatura, con un decremento de la población a mayor temperatura y acentuándose a partir de $\operatorname{los} 26{ }^{\circ} \mathrm{C}$. No se observó correlación con la humedad relativa. La mayor temperatura $\left(27.9^{\circ} \mathrm{C}\right)$ y humedad relativa $(88.2 \%)$ ocurrieron en el mes de julio y septiembre, respectivamente, meses en los cuales se observó la menor población de ácaros. Los acaricidas evaluados ejercieron un control significativo. A los 21 días después de la aplicación ocurrieron diferencias entre tratamientos y el testigo absoluto $(\mathrm{Pr}>\mathrm{F}=0.0245)$. Con la aplicación de azadiractina ( $1.9 \mathrm{~g}$ de ia $\mathrm{L}^{-1}$ de agua) y aceite mineral (16.9 $\mathrm{g}$ de ia $\mathrm{L}^{-1}$ de agua) se obtuvo un control de $75.9 \%$ y $72.5 \%$, respectivamente.

Palabras clave: Tetranychus pacificus Lam. (Moraceae), ácaros, control, yaca.

Recibido: marzo de 2019

Aceptado: junio de 2019 


\section{Introducción}

La yaca, Artocarpus heterophyllus Lam. (Moraceae), es originaria del suroeste de la India, actualmente se cultiva en países de Asia, África, América y El Caribe (Muniappan et al., 2012). En México su cultivo es reciente, con alrededor de 30 años de introducida a México ha tenido un crecimiento importante como producto de exportación (Ulloa et al., 2007; Luna et al., 2013). Con una superficie de 1509 ha, una producción anual de 22192 t cuyo valor asciende a poco más de 143.9 millones de pesos (SIAP, 2017), la yaca se ha convertido en un frutal importante y una opción de producción en zonas tropicales de Nayarit y Jalisco, México.

Debido a su reciente introducción y cultivo en México, la información sobre sus plagas y enfermedades asociadas y recomendaciones de manejo es aún limitada. Entre los escasos estudios sobre plagas se encuentra lo publicado por Rodríguez et al. (2017) quienes consignan algunas especies de chinches y piojos harinosos asociadas a yaca. Por su parte, Hernández et al. (2018), reportan por primera vez en Nayarit a Piezogaster odiosus (Stal.) y Leptopharsa sp., como insectos fitófagos de este cultivo. De igual manera la cochinilla rosada del hibisco (Maconellicoccus hirsutus Green) es considerada una plaga importante de la yaca en México (SENASICA, 2016). Al carecer de mayor información fitosanitaria, los técnicos y productores realizan recomendaciones y aplicaciones de plaguicidas de amplio espectro al observar daños al cultivo.

En la mayoría de los casos, se desconocen la identidad, biología, hábitos, distribución, fluctuación estacional y daños asociados a estas plagas. En este sentido en el año 2017 en Nayarit se observaron daños al follaje, deformación, decoloración del haz, y presencia de telaraña en el envés, causados posiblemente por el ácaro conocido como araña roja según reportes de los propios productores. Por lo anterior, los objetivos de la presente investigación fueron confirmar la presencia de araña roja y su identificación, determinar su distribución y fluctuación poblacional u su relación con temperatura y humedad relativa en Nayarit.

Además, con el fin de proporcionar una herramienta para los productores para el control de esta nueva plaga en yaca, se realizaron pruebas de eficacia biológica de varios acaricidas, la mayoría de ellos recomendados y aplicados en la actualidad por técnicos y productores, pero sin un sustento técnico, principalmente en el uso de la dosis y tipo de producto utilizado.

\section{Materiales y métodos}

\section{Identificación de la especie de araña roja observada}

Las muestras recolectadas de araña roja fueron enviadas al Centro Nacional de Referencia Fitosanitaria del Servicio Nacional de Sanidad, Inocuidad y Calidad Agroalimentaria para su identificación y se utilizó la descripción de Lee et al. (1985); Seeman y Beard (2011).

\section{Distribución, fluctuación poblacional y su relación con factores ambientales}

Los muestreos se hicieron en cuatro sitios ubicados en las principales zonas productoras de yaca en Nayarit. El clima predominante en la zona de acuerdo a García (2004) es cálido subhúmedo con lluvias en verano $\left(\mathrm{AW}_{1}\left(\mathrm{x}^{1}\right)\right.$. Con vegetación predominante de selva seca perennifolia $\mathrm{y}$ 
subcaducifolia (INEGI, 2018). Municipio de San Blas. Sitio 1. Jalcocotán, coordenadas: 21.2938 latitud norte, -105.0457 longitud oeste a 576 metros sobre el nivel medio del mar (msnm). Sitio 2. Tecuitata, coordenadas: 21.2626 latitud norte, -105.0857 longitud oeste a $252 \mathrm{msnm}$. Sitio 3 .

El llano, coordenadas: 21.2730 latitud norte, -105.1112 longitud oeste a $18 \mathrm{msnm}$. Municipio de Compostela. Sitio 4. El Capomo, coordenadas: 21.0613 latitud norte, -105.1035 longitud oeste a $103 \mathrm{msnm}$. En cada sitio de muestreo se colocó una micro estación WatchDog (Spectrum Technologies, Inc. Aurora, Illinois, EE. UU-) serie 1000 para el registro de humedad relativa y temperatura. Los muestreos se hicieron con base en lo sugerido por Southwood (1978) y Pedigo y Rice (2009) para estimaciones de la densidad poblacional por unidad de hábitat.

En cada sitio se eligió un huerto de entre ocho a diez años de edad con la mayor homogeneidad posible en cuanto a los fenotipos cultivados ya que se tienen varios materiales o selecciones consideradas como variedades (Luna et al., 2013) y aunque estas no están caracterizadas aún ni registradas en un catálogo, las diferencias fenotípicas son notables. Los muestreos se hicieron con una periodicidad de dos a tres semanas. Mediante muestreos previos se observó el daño de la araña roja en el envés y haz del follaje, no se observaron daños en flor o fruto. En cada sitio se eligieron al azar 30 hojas de cinco a diez árboles recorriendo el huerto en sentido aleatorio.

El tamaño de muestra se estimó según lo sugerido por Karandinos $(1976)$ : n= $(\mathrm{s} / E m)^{2}$, donde $\mathrm{n}=$ tamaño de muestra; $s=$ desviación estándar; $E=$ error estándar predeterminado (coeficiente de variabilidad de la media=0.2) y $m=$ media. El conteo fue directo en campo con una lupa 30x, se contabilizó el número de ácaros por hoja en ambos lados de esta. Se realizó una comparación de la media poblacional mensual entre los sitios muestreados y su relación con temperatura y humedad ambiental. Previo a su análisis estadístico, los datos de cada muestreo fueron transformados mediante la fórmula $\mathrm{x}=\sqrt{ }(\mathrm{y}+0.8)$, donde $\mathrm{y}=$ valor medio observado; lo anterior, con el fin de observar mayor normalidad de los datos y al mismo tiempo independizar la varianza de la media.

Se realizó una comparación de medias entre los sitios de muestreo (LSD, $\alpha=0.1$ ). Se calculó el coeficiente de regresión $(\beta)$ e índice de correlación de Pearson $(r)$ bajo los supuestos que fundamentan la regresión lineal, para determinar la relación de la fluctuación poblacional promedio de los sitios de muestreo (variable dependiente) entre la temperatura media $(\mathrm{tm})$ y humedad ambiental promedio $(H A P)$ (variables independientes) y con la interacción de estas $(t m * H A P)$, se utilizó el método de mínimos cuadrados (Daniel, 2011).

Asimismo, para determinar la significancia estadística de la linealidad $(r)$ entre la variable observada, se realizó la prueba estadística Fisher $(F)$ para comprobar $H_{0}$ : $\beta=0, H_{A}$ : $\beta \neq 0$ con $\alpha=0$. Se utilizó el programa de análisis estadístico SAS $^{\circledR}$ versión 9.3 (2010).

\section{Evaluación de eficacia biológica de acaricidas}

Se utilizaron productos acaricidas disponibles en el mercado local en la zona productora de yaca. El estudio se realizó dentro del huerto en el Sitio 2, Tecuitata, coordenadas: 21.2626 latitud norte, -105.0857 longitud oeste a $252 \mathrm{msnm}$. Las evaluaciones se hicieron de marzo a abril, se evaluaron los siguientes productos y sus dosis: 1) azufre (Intersul 725 $5^{\mathrm{TM}}$, Internacional Química de Cobre, SA de C. V.) $2.5 \mathrm{~mL} \mathrm{~L}^{-1}$ de agua; 2) abamectina (Hortimec $1.8^{\mathrm{TM}}$, Agroquimica Tridente, SA de 
CV) $1 \mathrm{~mL} \mathrm{~L}^{-1}$ de agua; 3) sales potásicas de ácidos grasos (Des-X $\mathrm{X}^{\mathrm{TM}}$, Summit Agro de México, SA de CV) $18 \mathrm{~mL} \mathrm{~L}^{-1}$ de agua; 4) extracto de nim (Trilogy ${ }^{\mathrm{TM}}$, Summit Agro de México, SA de CV) (3 $\mathrm{mL} \mathrm{L}^{-1}$ de agua; 5) aceite mineral (Akaroil ${ }^{\mathrm{TM}}$, Altiara) $\left(20 \mathrm{~mL} \mathrm{~L}^{-1}\right.$ de agua); y 6) testigo sólo con aplicación de agua.

Se realizó una aplicación con bomba motorizada Stihl ${ }^{\circledR}$ Mod. SR420, un gasto de agua de $1.2 \mathrm{~L}$ árbol $^{-1}$. Los tratamientos fueron distribuidos en bloques completos al azar con cinco repeticiones por tratamiento. Cada repetición consistió de dos árboles dejando una hilera sin aplicar entre repeticiones. Para contabilizar la población de araña roja, se muestrearon diez hojas por repetición elegidas al azar de la parte media de la copa del árbol. El conteo se realizó en campo con la ayuda de una lupa 30 x observando en ambos lados de las hojas. Se hizo un muestreo previo y cuatro muestreos posteriores a la aplicación de tratamientos.

Se realizó análisis de varianza y comparación de medias de tratamientos con Duncan $(\alpha=0.05)$ en cada fecha de muestreo después de la aplicación. Aunado a lo anterior, se comparó la eficacia biológica de cada tratamiento respecto al testigo absoluto en cada fecha de muestreo, se utilizó para ello la fórmula de Henderson y Tilton (1955).

\section{Resultados y discusión}

\section{Identificación de la especie}

La araña roja identificada es Tetranychus pacificus. La descripción de acuerdo a Lee et al. (1986); Seeman y Beard (2011) es: macho, estrías dorsales sin lóbulos. Empodio I uncinado, provisto de una fuerte espuela, el empodio II con espuela más pequeña y empodia II-IV con diminutas espuelas dorsales. Empodia II-IV presentan largos pelos proximoventrales y libres. Edeago sin protuberancia, sigmoide y presenta una proyección sobre el margen anterior. La coloración de ambos sexos depende del hospedante y la época del año, puede ser de color rojo intenso a café oscuro.

En el caso de lo encontrado en yaca sólo se han observado ácaros color rojo con diferentes tonalidades. Hasta nuestro conocimiento, este es el primer reporte de T. pacificus asociada a la $A$. heterophyllus. En otras especies del genero Artocarpus se han consignado otros ácaros fitófagos; al respecto, Flechtmann et al. (1999) mencionan a Paratetranychus biharensis Hirst asociado a Artocarpus altilis en Francia, mientras que Yusof y Zhang (2003) observaron esta misma especie en Artocarpus comminis en Malasia al igual que Tetranychus malaysiensis Ehara provocando daños en Artocarpus champeden. Por su parte Gutiérrez y Schicha (1983) reportan a Tetranychus ludeni Zacher como fitófago de Artocarpus incisa en Sidney, Australia. Lee (1986) reporta a Eutetranychus banksi McGregor de A. altilis en Maui, Oahu.

En la India, Gupta y Gupta (1994) mencionan a Panonychus citri McGregor, Panonychus ulmi Koch y Oligonychus mangiferus Rahman y Sapra asociada a Artocarpus integrifolia. Todos los miembros de Tetranychidae son fitófagos y constituyen un grupo muy importante de ácaros plaga en la agricultura, la mayoría de los daños son en el follaje y en fuertes infestaciones en frutos y tallos (NAPPO, 2014). T. pacificus inicia sus daños con puntos cloróticos en el haz, hasta llegar al bronceado total (Badii et al., 2010; Vacante, 2016). T. pacificus adicionalmente en la yaca puede provocar deformación de las hojas al dañarlas en etapas tempranas de su desarrollo. 
Al respecto, Vacante (2016) menciona daños severos causados por bajas poblaciones de $T$. pacificus en pera, cítricos y otros cultivos, sugiriendo que podría estar involucrada la inyección de toxinas al tejido vegetal. Es una seria plaga por su amplia distribución, desde zonas subtropicales cálidas como México hasta zonas templadas como Canadá (Seeman y Beard, 2011). El umbral de acción para $T$. pacificus es variable y depende de la susceptibilidad del hospedante, en vid por ejemplo 1200 ácaros hoja $^{-1} 4$ semanas $^{-1}$ no provocan daños en la producción, mientras que en almendra poblaciones altas reducen del 13-19\% el rendimiento de los árboles jóvenes en el siguiente año de producción (Badii et al., 2010).

Por ser un cultivo reciente y apenas detectado como plaga T. pacificus en yaca faltaría determinar el umbral de acción, daños directos e indirectos y cuantificar los daños en la producción. En uva los daños por T. pacificus inducen una reducción de azucares y perdidas en rendimiento (Vacante (2016). En maíz provocan secamiento prematuro del follaje lo cual es asociado a una reducción en el rendimiento (Jeppson et al., 1975). En sitios como Tecuitata se han llegado a observar árboles con síntomas de bronceado y con $100 \%$ del follaje dañado.

\section{Distribución, fluctuación poblacional y su relación con factores ambientales}

En los cuatros sitios muestreados se encontró T. pacificus en el cultivo de yaca en Nayarit (Cuadro 1). No obstante, la mayor población se ubica en la zona de Tecuitata y Jalcocotan en el municipio de San Blas. Las diferencias entre sitios son significativas (Cuadro 1), el mayor promedio anual ocurrió en Tecuitata con 0.86 ácaros hoja ${ }^{-1}$, seguido de Jalcocotan 0.57 ácaros hoja $^{-1}$. En ambos sitios la mayor población se observó en enero con 4.51 y 5.23 ácaros hoja ${ }^{-1}$, respectivamente.

Cuadro 1. Distribución y fluctuación poblacional de $T$. pacificus en Yaca. Nayarit, México. 2018-2019.

\begin{tabular}{cccccc}
\hline \multicolumn{6}{c}{ Ácaros hoja $^{-1}$} \\
\hline Mes & El Capomo & El Llano & Tecuitata & Jalcocotan & Promedio \\
\hline Febrero & 0 & 0.03 & 0.66 & 0.2 & 0.22 \\
Marzo & 0 & 0.06 & 0 & 0 & 0.02 \\
Abril & 0 & 0.1 & 0.96 & 0.46 & 0.38 \\
Mayo & 0 & 0 & 0.26 & 0.33 & 0.15 \\
Junio & 0 & 0.66 & 0 & 0 & 0.17 \\
Julio & 0.16 & 0 & 0 & 0.03 & 0.05 \\
Agosto & 0 & 0 & 0 & 0.53 & 0.13 \\
Septiembre & 0 & 0 & 0 & 0 & 0 \\
Octubre & 0 & 0 & 0 & 0 & 0 \\
Noviembre & 0 & 1.7 & 0 & 0 & 0.43 \\
Diciembre & 0 & 0.16 & 3.96 & 0 & 1.03 \\
Enero & 0 & 0 & 4.51 & 5.23 & 2.44 \\
Promedio & $0.01 \mathrm{~b}$ & $0.23 \mathrm{a}$ & $0.86 \mathrm{ab}$ & $0.57 \mathrm{ab}$ & 0.34 \\
\hline
\end{tabular}

* = medias con distinta letra son estadísticamente diferentes (LSD, $\alpha=0.1)$. 
El menor promedio anual se encontró en El Capomo con 0.01 ácaros hoja ${ }^{-1}$, solo se detectó en el mes de julio y El llano con 0.23 ácaros hoja ${ }^{-1}$. En esta región se inició el cultivo de la yaca hace poco más de 20 años (Luna et al., 2013) y es también donde se presenta la mayor superficie (SIAP, 2017). La temperatura media anual en los cuatro sitios de muestreo fue estadísticamente igual en El Capomo $\left(25.4^{\circ} \mathrm{C}\right)$, El Llano $\left(25.5^{\circ} \mathrm{C}\right)$ y Tecuitata $\left(25.1^{\circ} \mathrm{C}\right)(\mathrm{Pr}>\mathrm{F}=0.0231)$ y diferente en Jalcocotan $\left(22.7^{\circ} \mathrm{C}\right)$, con una temperatura mínima y máxima de $21.5^{\circ} \mathrm{C}, 22.2^{\circ} \mathrm{C}, 21.8^{\circ} \mathrm{C}, 19.3^{\circ} \mathrm{C}$ y $29.4{ }^{\circ} \mathrm{C}, 29.1^{\circ} \mathrm{C}, 28.3{ }^{\circ} \mathrm{C}, 24.9^{\circ} \mathrm{C}$, respectivamente. Sin embargo, la temperatura media anual de los cuatro sitios se encuentra en el rango óptimo para el desarrollo de T. pacificus según lo señalado por Badii et al. (2010).

En lo que respecta a la humedad ambiental anual, no se observaron diferencias significativas entre los sitios muestreados. Esta osciló en promedio de $76.9 \%$ en Jalcocotan a $79.8 \%$ en El Capomo. Con una máxima de $90.2 \%$ y una mínima de $46.2 \%$, ambas ocurridas en el sitio de Jalcocotan, del municipio de San Blas. La mayor población de ácaros ocurrió en los meses de enero a abril, habiendo un promedio general máximo en enero de 2.44 ácaros hoja ${ }^{-1}$ (4.51 y 5.23 ácaros/hoja en los sitios de Tecuitata y Jalcocotan, sitios más cercanos entre sí). A partir, de julio la población decrece hasta ser imperceptible en los meses de septiembre y octubre, periodo de lluvias y de mayor humedad en los sitios de muestreo.

El ciclo de vida de $T$. pacificus y por su relación directa de este con el incremento o decremento poblacional depende del hospedante, la temperatura y humedad ambiental; a una temperatura de 30-35 ${ }^{\circ} \mathrm{C}$ el tiempo de desarrollo de huevo a adulto de $T$. pacificus es de seis días, mientras que a $25^{\circ} \mathrm{C}$ es de 10-14 días (Badii et al., 2010). En general altas temperaturas y baja humedad ambiental favorecen el desarrollo de T. pacificus y sus daños asociados (Vacante, 2016). En otras especies como Olygonichus mangiferus la temperatura y humedad relativa óptimas son de $15-31{ }^{\circ} \mathrm{C}$ y 65 $75 \%$, respectivamente, condiciones fuera de estos rangos afectan significativamente su desarrollo, inhibiendo su crecimiento o provocando su muerte (Badawi et al., 2011).

Si bien el promedio poblacional anual de T. pacificus en los sitios muestreados es diferente estadísticamente, es probable que estas diferencias se deban, entre otras causas, al poco tiempo que se ha cultivado yaca en Nayarit, más que a factores ambientales ya que no existen grandes diferencias de temperatura y humedad, el cultivo de yaca inició en la región de San Blas (Sitios Jalcocotan, Tecuitata y El Llano) y se ha ido ampliando hacia otras zonas del estado (Sitio El Capomo) en los últimos diez años. Con el incremento de la superficie y distribución de yaca se incrementaría la distribución y abundancia de plagas.

\section{Correlación con temperatura y humedad ambiental}

En el caso de la relación densidad poblacional-humedad ambiental, no se observó una correlación significativa $(\beta=0.64, r=-0.1467, \mathrm{~F}=0.5572, \alpha=0.05)$ (Figura 1), por el contrario, con la temperatura se observa una correlación significativa $(\beta=0.0489, r=-0.736, \mathrm{~F}=0.0345, \alpha=0.05)$ (Figura 2) con un decremento de la población a mayor temperatura, acentuándose a partir de los 26 ${ }^{\circ} \mathrm{C}$ y hasta los $27.9{ }^{\circ} \mathrm{C}$ y no significativa con la interacción $(t m * H A P)(\mathrm{F}=0.1426, \alpha=0.05)$. La mayor temperatura $\left(27.9{ }^{\circ} \mathrm{C}\right)$ y humedad relativa $(88.2 \%)$ ocurrieron en el mes de julio y septiembre, respectivamente, meses en los cuales se observó la menor población de ácaros. 
Por el contrario, la menor temperatura $\left(21.2^{\circ} \mathrm{C}\right)$ y humedad relativa $(62 \%)$ se registraron en diciembre y abril, respectivamente, meses con mayor población de ácaros. No obstante, como se mencionó anteriormente, es probable que la distribución de araña roja en yaca en Nayarit esté en una etapa inicial de dispersión por el incremento en superficie y mayor distribución del cultivo.

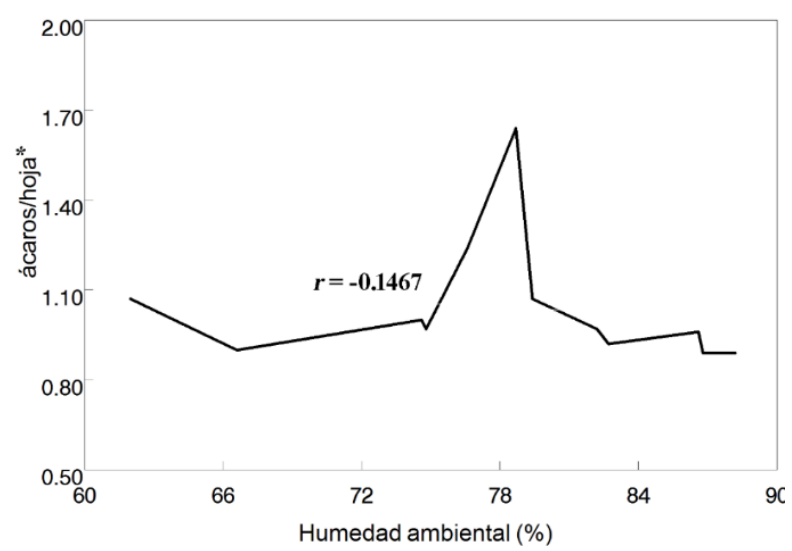

Figura 1. Fluctuación poblacional de $\boldsymbol{T}$. pacificus y su relación con humedad relativa en el cultivo de yaca A. heterophyllus. ${ }^{*}=$ datos transformados 2018-2019, Nayarit, México.

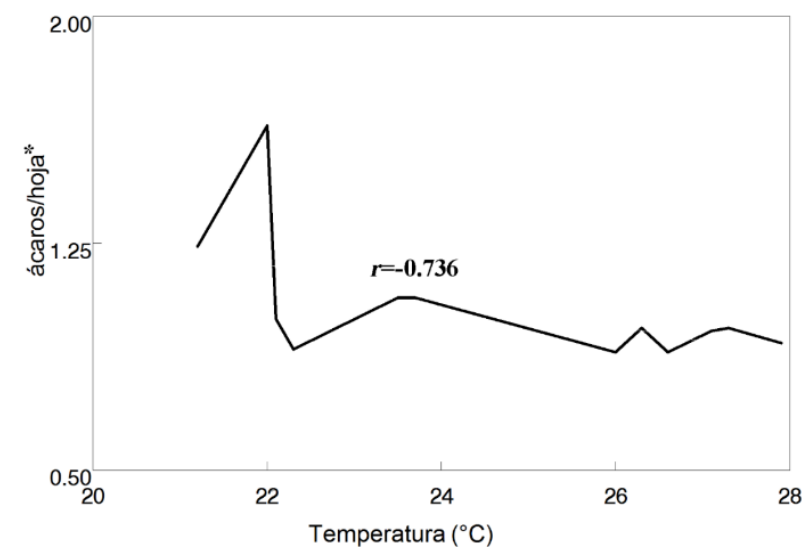

Figura 2. Fluctuación poblacional de $\boldsymbol{T}$. pacificus y su relación con temperatura en el cultivo de yaca A. heterophyllus. ${ }^{*}=$ datos transformados, 2018-2019, Nayarit, México.

\section{Evaluación de eficacia biológica de acaricidas}

El control de $T$. pacificus en el cultivo de yaca tuvo efectos estadísticos diferentes con los tratamientos evaluados (Cuadros 2 y 3). En el primer muestreo a los cinco días después de la aplicación, se observó una reducción importante de la población y ocurrieron diferencias estadísticas $(\mathrm{Pr}>\mathrm{F}=0.0056)$ respecto al testigo absoluto. Entre los tratamientos con acaricidas no se observaron diferencias significativas; sin embargo, el tratamiento con sales potásicas de ácidos grasos ejerció el mayor control con $95.4 \%$ de eficacia, le siguió la abamectina con $76.9 \%$ y el azufre con $76.2 \%$ de eficacia (Cuadro 2). 
Cuadro 2. Número promedio de ácaros $T$. pacificus en yaca después de la aplicación de tratamientos.

\begin{tabular}{|c|c|c|c|c|c|c|c|c|}
\hline \multirow{2}{*}{ Tratamiento } & \multirow{2}{*}{${ }^{1} \mathrm{~g}$ de ia $\mathrm{L}^{-1}$} & \multirow{2}{*}{$\begin{array}{c}{ }^{2} \text { Dosis (mL L } \mathrm{L}^{-1} \\
\text { de agua) }\end{array}$} & \multicolumn{6}{|c|}{${ }^{3}$ Medias de tratamientos } \\
\hline & & & Previo & $5 \mathrm{dda}$ & $8 \mathrm{dda}$ & $14 \mathrm{dda}$ & $21 \mathrm{dda}$ & $28 \mathrm{dda}$ \\
\hline 1) Azufre & 725 & 2.5 & $3.6 \pm 1$ & $0.7 b \pm 0.3$ & $0.1 c \pm 0.1$ & $1.6 b c \pm 0.4$ & $0.2 \mathrm{~b} \pm 0.1$ & $0.7 \pm 0.2$ \\
\hline 2) Abamectina & 18 & 1 & $3.2 \pm 0.7$ & $0.6 b \pm 0.3$ & $0.8 b c \pm 0.2$ & $1.6 b c \pm 0.4$ & $0.4 \mathrm{~b} \pm 0.2$ & $1.2 \pm 0.3$ \\
\hline $\begin{array}{l}\text { 3) Sales potásicas de } \\
\text { ácidos grasos }\end{array}$ & 582.8 & 18 & $4 \pm 0.2$ & $0.1 \mathrm{~b} \pm 0.04$ & $0.2 \mathrm{c} \pm 0.1$ & $0.3 c \pm 0.1$ & $0.1 b \pm 0.06$ & $0.3 \pm 0.1$ \\
\hline 4) Extracto de nim & 639.9 & 3 & $4.1 \pm 0.9$ & $1.5 b \pm 0.5$ & $1.8 \mathrm{~b} \pm 0.6$ & $1.4 b c \pm 0.5$ & $0.6 \mathrm{~b} \pm 0.4$ & $1.6 \pm 0.6$ \\
\hline 5) Aceite mineral & 847 & 20 & $3.3 \pm 0.7$ & $2.7 b \pm 1.2$ & $3.4 \mathrm{a} \pm 0.6$ & $4 a \pm 0.7$ & $0.1 b \pm 0.06$ & $0.5 \pm 0.2$ \\
\hline 6) Testigo absoluto & - & Sólo agua & $2.2 \pm 0.8$ & $6.5 \mathrm{a} \pm 2.1$ & $3.3 \mathrm{a} \pm 0.3$ & $3.3 \mathrm{a} \pm 0.8$ & $1.5 \mathrm{a} \pm 0.5$ & $1.4 \pm 0.6$ \\
\hline
\end{tabular}

${ }^{1}=$ gramos de ingrediente activo en producto comercial: ${ }^{2}=$ dosis de producto formulado ${ }^{3}=$ ácaros por hoja en días después de la aplicación (土error estándar).

Al repecto, Stavrinides y Mills (2009) evaluaron la eficacia de azufre (11.5 g de ia $\mathrm{L}^{-1}$ de agua) contra $T$. pacificus en el cultivo de vid y observaron una reducción significativa de su población, además mencionan que el azufre no tuvo efecto sobre su depredador Galendromus occidentalis Nesbitt. En la segunda evaluación, a los ocho días después de la aplicación, se observaron diferencias significativas $(\mathrm{Pr}>\mathrm{F}=0.0001)$ entre los tratamientos y el testigo absoluto. Los tratamientos con mayor eficacia en el control fueron el azufre y las sales potásicas de ácidos grasos con $100 \%$ y $82.3 \%$, respectivamente. En el tratamiento con aceite mineral no se observó efecto significativo respecto al testigo absoluto (Cuadro 2).

A los 14 días después de la aplicación la población de $T$. pacificus en los tratamientos fue estadísticamente diferente $(\mathrm{Pr}>\mathrm{F}=0.0035)$. El tratamiento con mayor control fue las sales potásicas de ácidos grasos con $92.4 \%$ de eficacia. El tratamiento con aceite mineral fue igual estadísticamente al testigo absoluto. A los 21 días después de la aplicación, ocurrieron diferencias significativas entre tratamientos y el testigo absoluto $(\mathrm{Pr}>\mathrm{F}=0.0245)$. Si bien entre los tratamientos las diferencias no fueron significativas, los tratamientos con mayor control fueron los de azadiractina y aceite mineral con $75.9 \%$ y $72.5 \%$ de eficacia, respectivamente (Cuadro 3).

Cuadro 3. Porciento de eficacia de control de $T$. pacificus en yaca después de la aplicación de tratamientos.

\begin{tabular}{lccccccc}
\hline \multirow{2}{*}{ Tratamiento } & \multicolumn{3}{c}{${ }^{1} \mathrm{~g}$ de ia $\mathrm{L}^{-1}{ }^{2}$ Dosis $\left(\mathrm{mL} \mathrm{L}^{-1}\right.$} & \multicolumn{5}{c}{ Porcentaje de eficacia de tratamientos } \\
& \multicolumn{1}{c}{ de agua) } & $5 \mathrm{dda}$ & $8 \mathrm{dda}$ & $14 \mathrm{dda}$ & $21 \mathrm{dda}$ & $28 \mathrm{dda}$ \\
\hline 1) Azufre & 725 & 2.5 & $76.2 \pm 19.1$ & $100 \mathrm{a} \pm 0$ & $49 \mathrm{ab} \pm 17.3$ & $65.9 \pm 19.3$ & $34.8 \pm 16.3$ \\
2) Abamectina & 18 & 1 & $76.9 \pm 19.3$ & $60.2 \mathrm{ab} \pm 17.8$ & $60.4 \mathrm{a} \pm 15.7$ & $42 \pm 21.2$ & $18.1 \pm 18.1$ \\
3) Sales potásicas de & 582.8 & 18 & $95.4 \pm 4.3$ & $82.3 \mathrm{a} \pm 17.7$ & $92.4 \mathrm{a} \pm 4.7$ & $40 \pm 24.4$ & $19.2 \pm 19.1$ \\
ácidos grasos & & & & & & & \\
4) Extracto de nim & 639.9 & 3 & $74.2 \pm 18.9$ & $69.5 \mathrm{a} \pm 9.7$ & $69.6 \mathrm{a} \pm 18.3$ & $75.9 \pm 19.2$ & $33.8 \pm 21.2$ \\
5) Aceite mineral & 847 & 20 & $64.6 \pm 19.7$ & $27 \mathrm{~b} \pm 13.6$ & $7.3 \mathrm{~b} \pm 7.2$ & $72.5 \pm 18.6$ & $43.6 \pm 18.1$ \\
6) Testigo absoluto & - & Sólo agua & - & - & - & - & - \\
\hline
\end{tabular}

${ }^{1}=$ gramos de ingrediente activo en producto comercial; ${ }^{2}=$ dosis de producto formulado; eficacia de control $( \pm$ error estándar); dda= días después de la aplicación. 
En la última evaluación a los 28 días después de la aplicación, la población de T. pacificus no fue diferente estadísticamente entre los tratamientos y el testigo absoluto. La mayor eficacia de control observada en esta evaluación fue en el tratamiento con aceite mineral con 43.6\% (Cuadro 3). Lo anterior, indica un periodo de efectividad con diferencias significativas de los tratamientos evaluados, con excepción del aceite mineral el cual ejerció un control irregular en las diferentes fechas de muestreo, de 21 días después de la aplicación con una eficacia de 40 a $75.9 \%$.

\section{Conclusiones}

La especie de araña roja asociada a la yaca en Nayarit es Tetranychus pacificus, reporte nuevo en este cultivo. Provoca daños al follaje, iniciando con punturas de color amarillo en el haz, deformación de éste y en altas infestaciones ocasiona una decoloración general tornándose amarillo-bronceado y provocando finalmente su caída. El daño severo ocasionado por bajas poblaciones en cultivos de pera, maíz, almendra, vid y cítricos la convierten en una plaga importante para la yaca. Su dispersión en Nayarit podría estar en etapa inicial, por lo que se debe considerar como plaga con potencial de causar dañosy tomar en cuenta para su manejo agronómico.

Con base a lo observado la fluctuación poblacional está relacionada principalmente con la temperatura. Se observa su presencia desde noviembre hasta agosto y es imperceptible en los meses de septiembre y octubre. La mayor población de araña roja se observó en diciembre y enero.

El control de $T$. pacificus en el cultivo de yaca tuvo efectos estadísticos diferentes con los tratamientos evaluados. A los cinco días después de la aplicación, se observó una reducción importante de la población y ocurrieron diferencias estadísticas respecto al testigo absoluto, el tratamiento con sales potásicas de ácidos grasos (10.4 g de ia $\mathrm{L}^{-1}$ de agua) ejerció el mayor control con $95.4 \%$ de eficacia, le siguió la abamectina $\left(0.018 \mathrm{~g}\right.$ de ia $\mathrm{L}^{-1}$ de agua) con $76.9 \%$ de eficacia.

A los 21 días después de la aplicación los tratamientos con mayor control fueron los de azadiractina

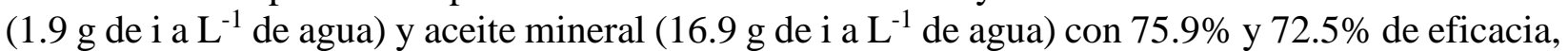
respectivamente.

Con estos resultados se logran los objetivos planteados; no obstante, son necesarios mayores estudios para un mejor entendimiento de la relación T. pacificus con Artocarpus heterophyllus. Se requieren estudios de biología, ciclos de vida, enemigos naturales asociados, tolerancia de fenotipos y su efecto en el rendimiento, además de evaluaciones de acaricidas con distinto mecanismo de acción y su impacto en los enemigos naturales. Todo lo anterior permitirá elaborar un mejor programa de manejo integrado de $T$. pacificus en el cultivo de yaca.

\section{Agradecimientos}

La presente investigación fue realizada con recursos del proyecto: diagnóstico, generación y transferencia de tecnología para el manejo fitosanitario sustentable del cultivo de yaca (Artocarpus heterophyllus) en México. Apoyado por el Fondo Sectorial SAGARPA-CONACYT en convocatoria: S0007-2017-02. Se agradece al Centro Nacional de Referencia Fitosanitaria por el apoyo para la identificación de la especie. Asimismo, se agradece la disponibilidad de los productores de yaca para realizar las observaciones en sus huertos. 


\section{Literatura citada}

Badawi A.; Abou, A.; Mahmoud, M.; Al, A. and Sahar, I. A. 2011. Effect of temperature and relative humidity on the rate of development, fecundity and lifetable parameters of the red spider mite Oligonychus mangiferus (Rahman and Sapra) (Acari:Tetranychidae). Alemania. Arch. Phytopathol. Plant Protection. 44(19):1862-1866.

Badii, M. H.; Landeros, J. y Cerna E. 2010. Regulación poblacional de ácaros plaga de impacto agrícola. México. Inter. J. Good Consci. 5(1):270-302.

Flechtmann, W. H. C.; Kreiter, S.; Etienne J. and De Moraes G. J. 1999. Plant mites (Acari) fo the French Antilles. 1. Tetranychoidea (Prostigamata). France. Acarologia. 50(2):137-144.

García, E. 2004. Modificaciones al sistema de clasificación climática de Köppen. $5^{\text {ta }}$. Edición. Instituto de Geografía-UNAM. México. 90 p.

Gupta, S. K. and Gupta, Y. N. 1994. A taxonomic review of Indian Tetranychidae (Acari: Prostigmata): Whit descriptions old new specie, re-descriptionsof known species, and keys to genera and species. India. Memoirs of the Zoological Survey of India. 18(1):1-196.

Gutiérrez, J. and Schicha, E. 1983. The spider mite family Tetranychidae (Acari) in New South Wales. United Kingdom. Inter. J. Acarol. 9(3):99-116.

Henderson, C. F. and Tilton, E. W. 1955. Tests with acaricides against the brow wheat mite. United States. J. Econ. Entomol. 48(2):157-161.

Hernández, F. L. M.; Brailovsky H. and Lopez M. V. 2018. Report of Piezogaster odiosus (Stal.) (Hemiptera: Coreidae) and Leptopharsa sp. (Hemiptera: Tingidae) as pests on Artocarpus heterophyllus Lam. Cultivated in Nayarit, Mexico. United States. Pan-Pacific Entomologist. 94(2):85-89.

INEGI. 2018. Instituto Nacional de Estadística, Geografía e Informática. Uso del suelo y vegetación. Instituto Nacional de Estadística, Geografía e Informática. http://www.beta.inegi.org.mx/temas/mapas/usosuelo/.

Jeppson, L. R.; Keifer, H. H. and Baker, E. W. 1975. Mites injurious to economic plants. University of California Press. United States. 614 p.

Karandinos, M. G. 1976. Optimum simple size and comments on some published formulae. United States. Bull. Ent. Soc. Am. 22:417-421.

Lee, G. M. 1986. Spider mites (Acari: Tetranychidae) in the Hawaiian Islands. United Kingdom. Inter. J. Acarol. 12(1):43-49.

Luna, E. G.; Alejo, S. G.; Ramírez, G. L. G. y Arévalo, G. M. L. 2013. La yaca (Artocarpus heterophyllus Lam.) un fruto de exportación. México. Agroproductividad. 6(5):65-70.

Muniappan, R.; Shepard, B. M.; Carner, G. R. and Aun, C. P. 2012. Arthropod pests of horticultural crops in Tropical Asia. CABI, Oxfordshire. $168 \mathrm{p}$.

NAPPO. 2014. Organización Norteamericana de Protección a las Plantas. Identificación morfológica de las arañas rojas (Tetranychidae) que afectan a las frutas importadas. Protocolos de Diagnóstico de la NAPPO. Ottawa, Ontario, Canadá. 36 p.

Pedigo, L. P. and Rice, M. E. 2009. Entomology and pest managment. $6^{\text {th }}$. Edición. Pearson Prentice Hall. Ohio, EUA. 784 p.

Rodríguez, P. M.; Cambero, C. J.; Luna, E. G.; Robles, B A. y Cambero K. G. N. 2017. Entomofauna asociada al cultivo de yaca (Artocarpus heterophyllus Lam.) en Nayarit, México. México. Entomología Mexicana. 4:220-223. 
Seeman, O. D. and Beard, J. J. 2011. Identification of exotic pest and Australian native and naturalised species of Tetranychus (Acari: Tetranychidae). New Zealand. Zootaxa. 2961(1):1-72.

SENASICA. 2016. Servicio Nacional de Sanidad Agroalimentaria, Acuícola y Pesquera. Cochinilla rosada del hibisco (M. hirsutus Green). Ficha técnica núm. 6. 26 p.

SIAP. 2017. Servicio de Información Agroalimentaria y Pesquera. https://nube.siap.gob.mx/ cierreagricola/.

Southwood, T. R. 1978. Ecological methods, with particular reference to the study of insect populations. Springer Netherlands. 2a ${ }^{\mathrm{a}}$. Edición. 548 p.

Stavrinides, M. C. and Mills, N. J. 2009. Demographic effects of pesticides on biological control of Pacific spider mite (Tetranychus pacificus) by the western predatory mite (Galendromus occidentalis). Amsterdan. Biol. Control. 48(3):267-273.

Ulloa, J. A.; Rosas, P.; Flores, J. R.; Ulloa, R. B. E. y Escalona, H. 2007. Comportamiento del color de bulbos del fruto de la yaca (Artocarpus heterophyllus) auto estabilizados en frascos de vidrio por la tecnología de obstáculos. España. Ciencia y Tecnología Alimentaria. 5(5):372-378.

Vacante, V. 2016. The handbook of mites of economic plants: Identification, bio-ecology and control. Ed. CABI. United Kingdom. 872 p.

Yusof, O. and Zhang, Z. Q. 2003. Tetranychidae (Acari: Prostigmata) of Malay Peninsula: Checklist, key to genera and species and description of three new species. New Zealand. Sistematic and Applied Acarology. 8(1):149-173. 